\title{
Potential role of exosome-based allorecognition pathways involved in lung transplant rejection
}

\author{
Billanna Hwang, DHSc, ${ }^{\text {a,b,c }}$ James Bryers, PhD, ${ }^{\text {b,c,d }}$ and Michael S. Mulligan, MD ${ }^{\mathrm{a}, \mathrm{b}, \mathrm{c}, \mathrm{e}}$
}

\begin{abstract}
Innate and adaptive immunity both contribute to allorecognition mechanisms that drive rejection after lung transplantation. Classic allorecognition pathways have been extensively described, but there continues to be several unanswered questions. Exosome research appears to be a novel and potentially significant area of allorecognition research and could be the missing link that answers some existing questions. This article reviews literature that is associated with allorecognition pathways and the role of exosomes in alloreactivity. (J Thorac Cardiovasc Surg 2021;161:e129-34)
\end{abstract}

From the Department of ${ }^{\text {a Surgery and }}{ }^{\mathrm{e}}$ Medicine, University of Washington School of Medicine, Seattle, Wash; ${ }^{\mathrm{b}}$ Center for Lung Biology and ${ }^{\mathrm{d}}$ Department of Bioengineering, University of Washington, Seattle, Wash; and ${ }^{\mathrm{c}}$ West Coast Exosortium (WestCo Exosortium), Seattle, Wash.

Research funding received from the National Institutes of Health (Bethesda, MD): Grant R01HL093097. The content is solely the responsibility of the authors and does not necessarily represent the official views of the National Institutes of Health or its subsidiary institutes and centers.

Received for publication Dec 21, 2018; revisions received April 2, 2020; accepted for publication April 9, 2020; available ahead of print June 18, 2020

Address for reprints: Billanna Hwang, DHSc, Department of Surgery, Center for Lung Biology, University of Washington, 850 Republican St, Box 358052, Seattle, WA 98109 (E-mail: bhwang@uw.edu).

J Thorac Cardiovasc Surg 2021;161:e129-34

$0022-5223 / \$ 36.00$

Copyright (C 2020 by The American Association for Thoracic Surgery

https://doi.org/10.1016/j.jtcvs.2020.04.183

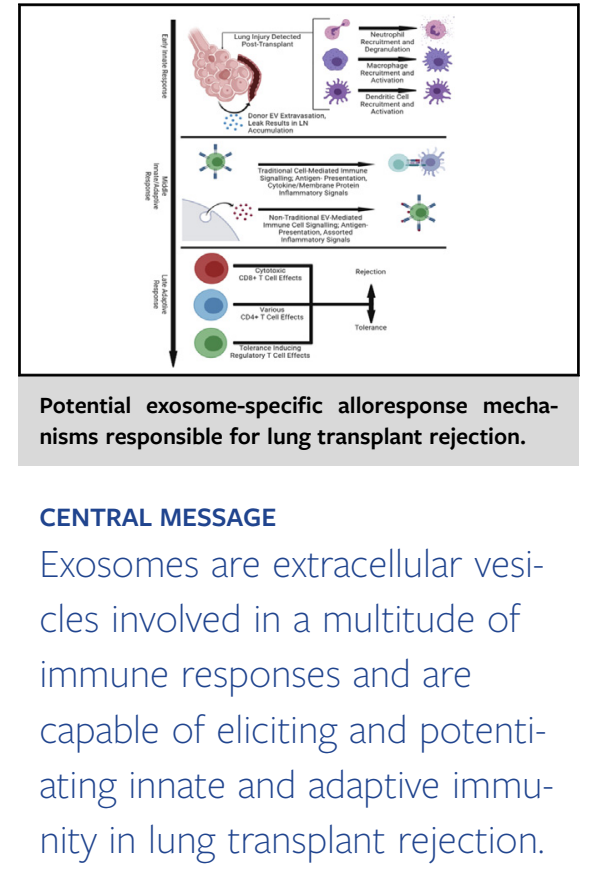

PERSPECTIVE
Classic allorecognition pathways have been
extensively described, but there continues to be
several unanswered questions. Exosome research
appears to be a novel and potentially significant
area of allorecognition research and could be
the missing link that answers some existing ques-
tions in lung transplant rejection.

See Commentaries on pages 135 and $\mathrm{e} 136$.

For end-stage lung disease, lung transplantation is the sole therapeutic option. ${ }^{1}$ Despite advances in the field, patients continue to face the worst post-transplant survival statistics. The half-life of a lung transplant is still limited to 5 years because of several complications, including acute and chronic allograft dysfunction (CLAD). ${ }^{1-3}$ Classically, immune mechanisms that drive the development of acute rejection and CLAD have been explained by mismatched donor human leukocyte antigens (HLAs) and HLAindependent lung-associated antigens. These immune responses are primarily dictated through T-cell:alloantigen responses, resulting in a series of immune cell signaling cascades that elicit activation of naïve cells after transplantation. ${ }^{3}$ In the area of alloimmunity, there are several implicated pathways describing T-cell:alloantigen interactions. 


\section{Abbreviations and Acronyms}

$\mathrm{APC}=$ antigen presenting cell

$\mathrm{BO}=$ bronchiolitis obliterans

$\mathrm{CLAD}=$ chronic allograft dysfunction

$\mathrm{DC}=$ dendritic cell

HLA = human leukocyte antigen

$\mathrm{MHC}=$ major histocompatibility complex

These pathways include direct, indirect, and semi-direct allorecognition. ${ }^{4-6}$ It has been classically accepted that at an early post-transplant stage, donor leukocytes migrate to recipient secondary lymphoid organs and activate naïve $\mathrm{T}$ cells in the direct pathway, generating alloreactive $\mathrm{T}$ cells that are mostly responsible for the development of acute rejection. As donor leukocytes diminish over time, the indirect pathway plays a major role in chronic rejection. ${ }^{3-5}$ Recipient antigen presenting cells (APCs) prime CD4+ T cells by internalizing and presenting allopeptides locally or in circulation. Later, it became evident that directly alloreactive $\mathrm{T}$ cells also recognize donor MHC molecules acquired intact by recipient APC through a third mechanism, the semi-direct pathway. The semi-direct pathway is also used to explain the cross-regulation between CD4 and CD8 T cells activated by distinct pathways. ${ }^{5}$ In the semi-direct pathway, the mechanism by which recipient APCs acquire and retain intact and functional donor MHC molecules is unclear. New evidence in the field of extracellular vesicles, especially exosomes, is currently providing new answers to these long-standing questions in the transplantation and allorecognition (Figure 1).

Exosomes are 30 to $100 \mathrm{~nm}$ extracellular vesicles released from the plasma membrane from an immune or nonimmune parent cell and can be isolated from various biological fluids including plasma, bronchioalveolar lavage, and urine. ${ }^{4-8}$ Exosome composition is dependent on the parent cell lineage, activation/differentiation states, and stages of inflammation and contain a variety of biologically active components including mRNA, micro-RNA, DNA, proteins, and alloMHC-peptide complexes. ${ }^{4-8}$ Exosomes are able to mediate immune responses for both innate and adaptive immunity through exosome:cell interactions. ${ }^{9-11}$ Characterization of exosomes through identifying surface markers and internal content, and correlating to functional capabilities are imperative to understanding their roles in transplant rejection. ${ }^{12-15}$ Most critical is to elucidate their roles in allorecognition pathways that drive acute and chronic rejection, and potentially redefine alloresponse paradigms at an exosome:cell level. In this review, we describe the classic allorecognition pathways that are involved in lung transplant rejection. In addition, we describe the potential roles of exosomes in allorecognition pathways or "allo-exoresponses," which provides a new and intriguing dynamic to the conventional thinking.

\section{ANTIGEN PRESENTING CELL PRODUCTION OF EXOSOMES OR "ALLO-EXO-ANTIGEN"}

Exosomes have been described to be secreted by various APC, including mast cells, ${ }^{9}$ natural killer cells, ${ }^{10}$ dendritic cells (DCs), macrophages, T cells, and B cells. ${ }^{16}$ Exosomes generated by APCs are highly enriched for surface MHCpeptide complexes, suggesting that they could function as antigen-presenting vesicles or as vehicles to disseminate allogeneic antigens for priming of anti-donor T cells. ${ }^{16,17}$ Exosomes generated by DCs have been observed in primary DCs and DC cell lines and have been the focus of many studies in the field of exosome-mediated allorecognition. ${ }^{16-19}$ Exosomes released by mature DCs are enriched for MHC, adhesion, and T-cell costimulatory molecules, home to lymphoid organs and prime T cells. ${ }^{18-20}$ Mature DCs also encounter secreted exosomes from various other cell subsets and present exo-allo-antigen to stimulate or inhibit cells. Montecalvo and colleagues ${ }^{18}$ described a short-range mechanism of alloantigen distribution through exosome delivery mechanisms to DCs, resulting in elicitation of the anti-donor $\mathrm{T}$ response observed in transplantation. It has also been demonstrated that exosomes are more important than soluble allopeptides in transferring antigen to DCs because antigens that are internalized and secreted in the form of phagosomes are presented $10^{3}$ - to $10^{4}$-fold more efficiently by MHC class II. ${ }^{18}$

Macrophages are known to play important roles in both innate immunity and adaptive immunity by phagocytosing pathogens, shaping innate responses, eliciting communication with adaptive immunity, and facilitating DC in antigen presentation. Exosomes isolated from macrophages have been found to enhance CD4+ and CD8+ T-cell responses particularly in the presence of DCs. ${ }^{21}$ In a mouse model of Mycobacterium tuberculosis infection, mice with Rab27a deficiency were found to be less efficient at stimulating $\mathrm{T}$ cells as a result of compromised capacity to release exosomes. $^{22}$

\section{ALLO-EXO-RECOGNITION PATHWAYS}

After transplantation, donor antigens can be acquired by APCs residing within the lung and lymphoid tissues (monocytes, macrophages, DCs, B cells, epithelial cells) through various mechanisms, including phagocytosis of dead cells, endocytosis, or micropinocytosis and secreted in the form of exosomes (exo-alloantigens). ${ }^{23-27}$ Exoalloantigens are released by leukocytes and have the potential to activate surrounding resident and infiltrating cells, as well as stimulate DC presentation within the 


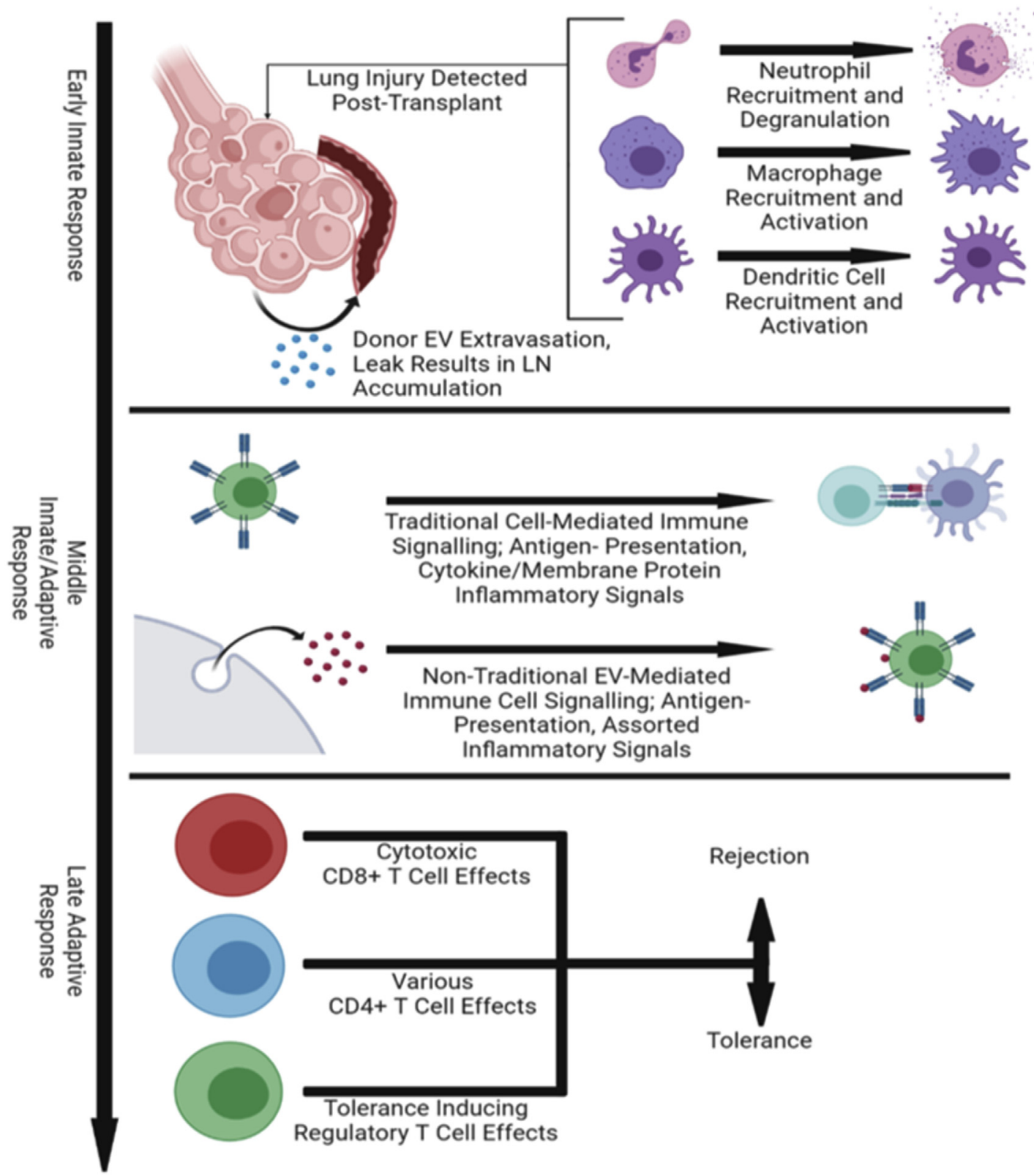

FIGURE 1. Exosome-specific alloresponse mechanisms. Exosomes play a major role in major immune stages after lung transplantation that dictate rejection or tolerance of the allograft. Early innate immune response mediate lung injury post-transplant where donor EVs "leak" out of the transplantation lung and travel through the bloodstream to nearby lymph nodes. These EVs participate in allomechanisms that elicit inflammatory responses, which develops in the late adaptive response through activation of T cells. $E V$, exosomes; $L N$, lymph nodes.

lymphoid tissues (Figure 1). This process is facilitated by recipient DCs cross-dressed with donor exosomes displaying intact donor MHC-peptide complexes. Although a majority of research investigating exosomes as a biomarker for rejection has been performed in animal models, most recently, a few clinically based studies have demonstrated the feasibility and identification of potential exosomebased biomarkers in lung transplant recipients. Gunasekaran and colleagues ${ }^{28,29}$ identified exosomes in circulation containing donor HLA and lung-associated self-antigens have been detected and characterized after lung transplantation. Levels of these self-antigen exoallo-antigens were associated with an increased incidence of acute and chronic rejection. ${ }^{28,29}$ Of note, acute rejection episodes have also been observed in nonvascularized allografts in which donor leukocytes are unable to migrate out of the transplanted tissue. Previous studies show that graftderived exosomes can travel across lymphatic endothelium 
in vitro and can be transported within lymphatic vessels in vivo. ${ }^{30}$ It appears that donor-derived exosomes "leak out" of the graft and travel through extravasation or severed openings of recipient lymphatic capillaries toward the graft-draining lymphoid organs. ${ }^{31}$

\section{Direct Allo-exo-recognition}

The direct allorecognition pathway explains how recipient $\mathrm{T}$ cells in the lymphoid organs recognize the intact donor peptide-major histocompatibility complexes (MHCs) displayed on the surface of donor APCs. Passenger donor APCs travel to recipient secondary lymphoid organs, resulting in the development of alloreactive $\mathrm{T}$ cells with a wide range of receptor specificities. The persistence of these alloresponse signaling cascades ultimately leads to the development of acute and chronic rejection in patients. In a kidney rat transplant model, prolonged allograft survival was achieved by the depletion of donor DCs, and tolerance of the allograft was prevented by repletion of donor DCs, ${ }^{32}$ demonstrating the importance of direct allorecognition in the development of tolerance or rejection.

Although several studies demonstrate the importance of donor DCs, many groups have reported that donor-derived DCs are undetectable in graft-draining lymphoid organs during the first week after transplantation of nonvascularized or vascularized allografts, ${ }^{33,34}$ suggesting there may be other mechanisms in facilitating the direct allorecognition. Recent evidence indicated that exosomes may enhance the efficiency of allo-sensitization of $\mathrm{T}$ cells against donor MHC molecules in the direct pathway. Exosomes maintain the topology of the APC of origin, exposing the extracellular domain of MHC molecules at the vesicle surface. Exosomes released by APCs carry surface MHC class I and MHC class II of parent cells, and potentially have capacities to directly stimulate CD8 and CD4 T cells. ${ }^{33}$

\section{Indirect Allo-exo-recognition}

By the indirect pathway, alloantigens are internalized by recipient APCs, processed and presented as peptide by selfMHC class II molecules to CD4+ T cells. ${ }^{34,35}$ This mechanism has been described to be persistent throughout the duration of the allograft and is considered to be the major contributor of chronic graft rejection, which is dominated by $\mathrm{CD} 4+\mathrm{T}$ cells and the generation of alloreactive antibodies. Patients with bronchiolitis obliterans (BO) show significantly higher frequencies of indirect donor alloreactivity compared with patients without $\mathrm{BO} .^{36}$ Assessment of a direct alloresponse in $\mathrm{T}$ cells showed less reactivity and a presence in both patients with or without BO. These data, along with several other groups, demonstrate that indirect allorecognition pathways are associated more with the development of chronic rejection. ${ }^{28,29,34-36}$ Although free exosomes have limited ability to induce alloreactive $\mathrm{T}$ cells directly, the p-MHC complexes carried by them can be used as a source of peptide to indirectly prime $\mathrm{T}$ cells when internalized by APCs through phagocytosis or micropinocytosis. ${ }^{37}$ Robbins and Morelli ${ }^{7}$ demonstrated that exosomes carrying allopeptide could trigger specific CD4 T-cell responses in wild-type mice, but not in MHC class II-deficient hosts. ${ }^{7}$

\section{Semi-direct Allo-exo-recognition}

The semi-direct pathway occurs when intact donor MHC molecules are transferred from donor to recipient DCs and subsequently presented by recipient DCs to $\mathrm{T}$ cells. ${ }^{38}$ This mechanism enables the cross-regulation between directly and indirectly activated $\mathrm{T}$ cells through the same APC. Lechler and Batchelor ${ }^{32}$ demonstrated that recipient-derived, conventional DCs cross-dressed with allogeneic donor MHC molecules could activate allospecific $\mathrm{T}$ cells, setting the basis of the semi-direct pathway of T-cell allorecognition in transplantation. Later, the presence of donor MHC molecules on recipient APCs was confirmed in both solid organ and allogeneic bone marrow transplantation. ${ }^{39,40}$

A recent study suggests the involvement of exosomes in the semi-direct pathway based on super-structural observations. ${ }^{33}$ Post-skin and heart allograft transplantation, recipient-derived DCs and B cells in the graft-draining lymphoid organs were cross-dressed with donor-derived exosomes, carrying intact donor MHC molecules. ${ }^{31,33}$ Of note, rather than being internalized by recipient DCs as previously described, exosomes adhered to the surface of recipient DCs in small clusters, retaining intact and functional donor MHC molecules and APC-activating signals. The depletion of these DCs drastically impaired T-cell priming against donor $\mathrm{MHC}$ and delayed cardiac allograft rejection. ${ }^{33}$ The semi-direct allo-exo-recognition pathway can give rise to a form of "split tolerance." ${ }^{41-44}$ This process is observed during chronic allo-exo exposure due to indirect alloreactive T-helper cells and direct alloreactive $\mathrm{T}$ effectors differentially affected by host DCs after exposure to "cross-dressed" exosomes. ${ }^{45}$

\section{Innate Allo-exo-recognition}

Traditionally, innate immunity has been described as a short-lived, rapid, nonspecific immune response to pathogens by macrophages, natural killer cells, and neutrophils. Most recently, this concept has been challenged, more specifically describing a potential role in determining nonself in tissues and cells in a nonmicrobial setting through an innate allorecognition pathway. ${ }^{43}$ Recent studies have shown allospecific responses in macrophages contribute to the process of rejection. ${ }^{46,47}$ In a model of T-cell-specific allorecognition restricted to a single foreign antigen, rejection was only observed when allogeneic nonself signals were recognized by host innate immunity. ${ }^{48}$ Additionally, mice deficient in T cells, B cells, and innate lymphoid cells transplanted with allogeneic grafts were still able to 
persistently differentiate monocytes into mature DCs. ${ }^{46,47}$ This could explain the role of innate immunity in triggering or enhancing graft rejection through maturation of host monocyte-derived DC function to activate $\mathrm{T}$ cells toward an effector phenotype and inducing allotoxicity activity in macrophages. ${ }^{46}$ The role of exosomes in innate allo-exorecognition has yet to be fully investigated, and there are several areas that exosomes could play a critical role. These include macrophage phagocytosis and antigen presentation, maturation of infiltrating monocytes toward an effector DC phenotype within the graft, and secretion of alloreactive, donor-specific exosomes that migrate to host lymphoid tissues for further antigen processing (Figure 1). ${ }^{46,47}$ Recent studies have revealed a potential mechanism based on a polymorphic molecule, signal regulatory protein $\alpha$ that exists on donor monocytes and secreted exosomes, and appears to contribute to donor:recipient alloreactivity. ${ }^{49}$

Innate exosome-based allorecognition could be the missing link in the development of chronic rejection due to the persistence of donor-specific exosomes residing in lymphoid tissues long after transplant.

\section{CONCLUSIONS}

Exosome-mediated allorecognition involves both innate and adaptive immunity. There have been numerous studies investigating the roles of direct, indirect, and semi-direct pathways in allorecognition driving transplant rejection. Further studies investigating donor or host-derived exosomes locally or in the secondary lymphoid organs in exosome-mediated allorecognition may provide new perspectives in addressing outstanding questions in the area of transplantation. The addition of potential exo-allorecognition pathways to the conventional theories provides significant insight into other mechanisms contributing to donor and recipient alloreactivity. The clearance of donor passenger leukocytes, or inability to migrate to recipient lymphoid tissues, suggests that donor graft-derived exosomes play a significant role in alloantigen recognition pathways. Although the mechanisms in which exosomes mediate acute and chronic rejection are still unclear, further studies investigating these pathways are pivotal to understanding the development of these immune processes and will provide insight for future therapeutics and identification of biomarkers for better patient outcomes.

\section{Conflict of Interest Statement}

The authors reported no conflicts of interest

The Journal policy requires editors and reviewers to disclose conflicts of interest and to decline handling or reviewing manuscripts for which they may have a conflict of interest. The editors and reviewers of this article have no conflicts of interest.

\section{References}

1. Arcasoy SM, Kotloff RM. Lung transplantation. N Engl J Med. 1999;340: 1081-91.

2. Trulock EP, Christie JD, Edwards LB. Registry of the International Society for Heart and Lung Transplantation: twenty-fourth official adult lung and heartlung transplantation report-2007. J Hear Lung Transplant. 2007;26:782-95.

3. Grossman EJ, Shilling RA. Bronchiolitis obliterans in lung transplantation: the good, the bad, and the future. Transl Res. 2009;153:153-65.

4. Lin CM, Gill RG. Direct and indirect allograft recognition: pathways dictating graft rejection mechanisms. Curr Opin Organ Transplant. 2016;21(1):40-4.

5. Afzali B, Lombardi G, Lechler RI. Pathways of major histocompatibility complex allorecognition. Curr Opin Organ Transplant. 2008;13:438-44.

6. Benichou G, Wang M, Ahrens K, Madsen JC. Extracellular vesicles in allograft rejection and tolerance. Cell Immunol. 2020;349:104063.

7. Robbins PD, Morelli AE. Regulation of immune responses by extracellular vesicles. Nat Rev Immunol. 2014;14:195-208.

8. Morelli AE, Baran-Bracamonte W, Burlingham WJ. Donor-derived exosomes: the trick behind the semi-direct pathway of allorecognition. Curr Opin Organ Transplant. 2017;22:46-54.

9. Skokos D, Le Panse S, Villa I. Mast cell-dependent B and T lymphocyte activation is mediated by the secretion of immunologically active exosomes. J Immunol. 2001;166:868-76.

10. Lugini L, Cecchetti S, Huber V. Immune surveillance properties of human NK cell-derived exosomes. J Immunol. 2012;189:2833-42.

11. Théry C, Duban L, Segura E, Véron P, Lantz O, Amigorena S. Indirect activation of naïve CD4+ T cells by dendritic cell-derived exosomes. Nat Immunol. 2002; 3:1156-62.

12. Bansal S, Sharma M, Ranjithkumar R, Mohanakumar T. The role of exosomes in allograft immunity. Cell Immunol. 2018;331:85-92.

13. Habertheuer A, Korutla L, Rostami S, Reddy S, Lal P, Naji A, et al. Donor tissuespecific exosome profiling enables noninvasive monitoring of acute rejection in mouse allogeneic heart transplantation. J Thorac Cardiovasc Surg. 2018;155:2479-89.

14. Gonzalez-Nolasco B, Wang M, Prunevieille A, Benichou G. Emerging role of exosomes in allorecognition and allograft rejection. Curr Opin Organ Transplant. 2018;23:22-7.

15. Sukma Dewi I, Celik S, Karlsson A, Hollander Z, Lam K, McManus JW, et al Exosomal miR-142-3p is increased during cardiac allograft rejection and augments vascular permeability through down-regulation of endothelial RAB11FIP2 expression. Cardiovasc Res. 2017;113:440-52.

16. Lindenbergh MFS, Stoorvogel W. Antigen presentation by extracellular vesicles from professional antigen-presenting cells. Annu Rev Immunol. 2018;36:435-9.

17. Kowal J, Arras G, Colombo M. Proteomic comparison defines novel markers to characterize heterogeneous populations of extracellular vesicle subtypes. Proc Natl Acad Sci. 2016;113:E968-77.

18. Montecalvo A, Shufesky WJ, Beer Stolz D. Exosomes as a short-range mechanism to spread alloantigen between dendritic cells during $\mathrm{T}$ cell allorecognition. J Immunol. 2008;180:3081-90.

19. Segura E, Nicco C, Lombard B. ICAM-1 on exosomes from mature dendritic cells is critical for efficient naive T-cell priming. Blood. 2005;106:216-23.

20. Allan RS, Waithman J, Bedoui S. Migratory dendritic cells transfer antigen to a lymph node-resident dendritic cell population for efficient CTL priming. Immunity. 2006;25:153-62.

21. Backer R, Schwandt T, Greuter M. Effective collaboration between marginal metallophilic macrophages and CD8+ dendritic cells in the generation of cytotoxic T cells. Proc Natl Acad Sci U S A. 2010;107:216-21.

22. Smith VL, Cheng Y, Bryant BR, Schorey JS. Exosomes function in antigen presentation during an in vivo Mycobacterium tuberculosis infection. Sci Rep. 2017; $7: 43578$.

23. Cunningham AC, Zhang JG, Moy JV, Ali S, Kirby JA. A comparison of the antigen-presenting capabilities of class II MHC-expressing human lung epithelial and endothelial cells. Immunology. 1997;91:458-63.

24. Van Niel G, Mallegol J, Bevilacqua C. Intestinal epithelial exosomes carry MHC class II/peptides able to inform the immune system in mice. Gut. 2003;52:1690-7.

25. Fukami N, Ramachandran S, Takenaka M, Weber J, Subramanian V, Mohanakumar T. An obligatory role for lung infiltrating B cells in the immunopathogenesis of obliterative airway disease induced by antibodies to MHC class I molecules. Am J Transplant. 2012;12:867-76.

26. Liu Q, Rojas-Canales DM, Divito SJ, Shufesky WJ, Stolz DB, Erdos G, et al Donor dendritic cell-derived exosomes promote allograft-targeting immune response. J Clin Invest. 2016;126:2805-20. 
27. Raposo G. B lymphocytes secrete antigen-presenting vesicles. J Exp Med. 1996; 183:1161-72.

28. Gunasekaran M, Xu Z, Nayak DK. Donor-derived exosomes with lung self-antigens in human lung allograft rejection. Am J Transplant. 2017;17: 474-84.

29. Gunasekaran M, Sharma M, Hachem R, Bremner R, Smith MA, Mohanakumar T. Circulating exosomes with distinct properties during chronic lung allograft rejection. J Immunol. 2018;200:2535-41.

30. Srinivasan S, Vannberg FO, Dixon JB. Lymphatic transport of exosomes as a rapid route of information dissemination to the lymph node. Sci Rep. 2016;6: 24436.

31. Marino J, Babiker-Mohamed MH, Crosby-Bertorini P. Donor exosomes rather than passenger leukocytes initiate alloreactive $\mathrm{T}$ cell responses after transplantation. Sci Immunol. 2016;1:aaf8759.

32. Lechler RI, Batchelor JR. Restoration of immunogenicity to passenger celldepleted kidney allografts by the addition of donor strain dendritic cells. $J$ Exp Med. 1982;155:31-41.

33. Zhuang Q, Liu Q, Divito SJ, Zeng Q, Yatim KM, Hughes AD, et al. Graftinfiltrating host dendritic cells play a key role in organ transplant rejection. Nat Commun. 2016;7:12623.

34. Fangmann J, Dalchau R, Fabre JW. Rejection of skin allografts by indirect allorecognition of donor class I major histocompatibility complex peptides. $J$ Exp Med. 1992;175:1521-9.

35. Bradley JA. Indirect $\mathrm{T}$ cell recognition in allograft rejection. Int Rev Immunol. 1996; 13:245-55.

36. Stanford RE, Ahmed S, Hodson M, Banner NR, Rose ML. A role for indirect allorecognition in lung transplant recipients with obliterative bronchiolitis. Am J Transplant. 2003;3:736-42.

37. Mallegol J, Van Niel G, Lebreton C. T84-intestinal epithelial exosomes bear MHC class II/peptide complexes potentiating antigen presentation by dendritic cells. Gastroenterology. 2007;132:1866-76.

38. Herrera OB, Golshayan D, Tibbott R. A novel pathway of alloantigen presentation by dendritic cells. J Immunol. 2004;173:4828-37.
39. Brown K, Sacks SH, Wong W. Extensive and bidirectional transfer of major histocompatibility complex class II molecules between donor and recipient cells in vivo following solid organ transplantation. FASEB J. 2008;22: 3776-84.

40. Markey KA, Koyama M, Gartlan KH. Cross-dressing by donor dendritic cells after allogeneic bone marrow transplantation contributes to formation of the immunological synapse and maximizes responses to indirectly presented antigen. $J$ Immunol. 2014;192:5426-33.

41. Kotloff RM, Ahya VN, Crawford SW. Pulmonary complications of solid organ and hematopoietic stem cell transplantation. Am J Respir Crit Care Med. 2004; 170:22-48.

42. Busch A, Quast T, Keller S. Transfer of T cell surface molecules to dendritic cells upon CD4+ T cell priming involves two distinct mechanisms. J Immunol. 2008; 181:3965-73.

43. Palmer SM, Burch LH, Davis RD. The role of innate immunity in acute allograft rejection after lung transplantation. Am J Respir Crit Care Med. 2003;168: 628-32.

44. Bracamonte-Baran W, Florentin J, Zhou Y, Jankowska-Gan E, Haynes WJ, Zhong W, et al. Modification of host dendritic cells by microchimerismderived extracellular vesicles generates split tolerance. Proc Natl Acad Sci U S A. 2017;114:1099-104.

45. Zeng F, Morelli AE. Extracellular vesicle-mediated MHC cross-dressing in immune homeostasis, transplantation, infectious diseases, and cancer. Semin Immunopathol. 2018;40:477-90.

46. Lakkis FG, Li XC. Innate allorecognition by monocytic cells and its role in graft rejection. Am J Transplant. 2018;18:289-92.

47. Chow KV, Delconte RB, Lew AM. Innate allorecognition results in rapid accumulation of monocyte-derived dendritic cells. J Immunol. 2016;197: 2000-8.

48. Uehara H, Minami K, Tullius SG. Recall features and allorecognition in innate immunity. Transpl Int. 2018;31:6-13.

49. Koh E, Lee EJ, Nam GH, Kim IS. Exosome-SIRP $\alpha$, a CD47 blockade increases cancer cell phagocytosis. Biomaterials. 2017;121:121-9. 